\title{
FRANÇOIS DE CONZIÉ (1356-1431) UND DIE POLITIK DER KURIE IN AVIGNON BIS ZUM KONZIL VON PISA
}

Die Beziehungen zwischen regnum und imperium wurden durch das Große Abendländische Schisma schwer belastet. Frankreich begab sich in die Obödienz der Avignoneser Päpste Clemens VII. und Benedikt XIII., während das Imperium auf seiten der römischen Obödienz verblieb. Jedoch waren sowohl der französische König als auch der römisch-deutsche Kaiser an einem Ende der Kirchenspaltung interessiert.

In den komplizierten und langwierigen Verhandlungen, die dem Ausbruch des Schismas folgten, spielte François de Conzié, Kämmerer der Avignoneser Obödienz, eine bedeutende Rolle; er soll im Mittelpunkt dieser Studie stehen.

Zur Person: Als jüngerer Sohn Pierres III., des Herrn (seigneur) von Conzié, und der Ancelise de Verbouz wurde François de Conzié im Jahre 1356 geboren. Aus der "Gallia Christiana« erfahren wir, daß er aus dem Bugey, einer Herrschaft der Grafen von Savoyen, stammte ${ }^{1}$. Dort finden wir noch heute das Dorf Conzieu (im November 2003 hatte es 101 Einwohner) und es ist wahrscheinlich, daß der Familienname sich von dieser Ortschaft ableitet. Das Geschlecht derer von Conzié war demnach eine Vasallenfamilie der Grafen von Savoyen ${ }^{2}$.

Für François de Conzié war eine kirchliche Laufbahn vorgesehen. Dennoch erhielt er seine erste Erziehung nicht in einem Kloster, sondern am savoyischen Hof in Chambéry, höchstwahrscheinlich ab 1362/1363, zusammen mit seinem älteren Bruder Jean ${ }^{3}$.

${ }^{1}$ Gallia Christiana in provincias distributa, hg. von Dionysius SAMMARTHANUS, neu hg. von Paulus Piolin, Bd. 13, Paris 1774, S. 43.

${ }^{2}$ Louis BINZ, Le népotisme de Clément VII et le diocèse de Genève, in: Genèse et débuts du Grand Schisme d'Occident, Paris 1980, S. 107-123, hier S. 114. Michel Hayez rechnet Conzié irrigerweise zu den Vasallen der Grafen von Genf. Vgl. Michel HAYEZ, Conzié, in: Lexikon des Mittelalters, Bd. 3, München 1986, Sp. 212f.

${ }^{3}$ Gallia Christiana (wie Anm. 1), S. 43: Ab ineunte atate omni scientiarum genere institutus in curia Amadei VI. Sabaudice comitis [...]; Roger Ch. LOGOZ, Clément VII (Robert de Genève): Sa chancellerie et le clergé romand au début du grand schisme (1387-1394), Lausanne 1974, S. 8. Quellen zu seinen eigenen Lehrjahren sind nicht auffindbar. 
Allem Anschein nach war er bereits im Jahre 1379 Doktor beider Rechte ${ }^{4}$. An welcher Universität er jedoch seine Studien absolvierte, wissen wir nicht, wenn auch eine gewisse Wahrscheinlichkeit für die Universität von Orléans spricht ${ }^{5}$.

$\mathrm{Da} ß$ Conzié an der Kurie Beschäftigung fand, wird meist mit seinen verwandtschaftlichen Beziehungen zu den Grafen von Savoyen und den Grafen von Genf erklärt, mit denen auch Clemens VII. verwandt war. Eine Schlüsselstellung kam hier dem päpstlichen Kämmerer zu, da er für die Einstellung des kurialen Personals zuständig war. Pierre de Cros, päpstlicher Kämmerer schon unter Gregor XI., war nach dem Ausbruch des Schismas 1378 damit beschäftigt, die Einheit der Kammer und eine effiziente Arbeitsweise wiederherzustellen. Er benötigte fähige Mitarbeiter und mag in dem ungefähr 22 Jahre alten Doktor beider Rechte einen geeigneten Kandidaten gesehen haben. Der Kämmerer hatte Conzié wahrscheinlich schon früher kennengelernt, als er mit Graf Amadeus VI. von Savoyen verhandelt hatte, um mit diesem und dem Markgrafen von Montferrat ein Bündnis gegen Mailand zustande zu bringen. Jedenfalls wurde Conzié 1378 von Pierre de Cros zum Auditor ernannt ${ }^{6}$. Als Conziés Verwandter, Rodolphe de Chissé, Bischof von Grenoble, das Erzbistum Tarantaise erhielt, wurde Conzié 1380 durch Clemens VII. zum Nachfolger Rodolphes in Grenoble bestimmt ${ }^{7}$. Am 23. Dezember 1383 ernannte Clemens VII. Pierre de Cros zum Kardinal. Es war nicht üblich, daß ein Kardinal zugleich Kämmerer war, daher mußte ein Nachfolger gefunden werden. Neuer Kämmerer wurde am 24. Dezember 1383 François de Conzie ${ }^{8}$. Damit wurde Conzié das wohl wichtigste Amt an der Kurie übertragen. Der päpstliche Kämmerer war sowohl Vorsteher des päpstlichen Haushalts als auch der päpstlichen Finanzverwaltung. Er allein kannte alle Einnahmequellen, er allein

${ }^{4}$ So übereinstimmend die Literatur. Einen eindeutigen Quellenbeleg habe ich jedoch nicht gefunden.

${ }^{5}$ Conzié erscheint 1379 als Kanoniker von Chartres, ein Benefizium, das er sicherlich schon während seiner Studienzeit besaß. Sucht man nach Universitäten im näheren geographischen Umfeld, so kommen Paris und Orléans in die nähere Auswahl. Eine gewisse Wahrscheinlichkeit spricht für Orléans. Zwar genoß Paris das höchste Ansehen unter den französischen Universitäten, indes war Orléans besonders bei Ausländern sehr beliebt. Auch war Conzié in späteren Jahren ein zäher Gegner der Universität von Paris; sicherlich hätte er sich nicht gegen seine ehemalige Alma Mater gestellt.

${ }^{6}$ Michel HAYEZ, Pierre (II) de Cros, in: Lexikon des Mittelalters, Bd. 3, München 1986, Sp. $356 f$.

${ }^{7}$ T. MOREMBERT, Conzié 1.-3., in: Dictionnaire de biographie française, hg. von Roman D'AMAT, Bd. 9, Paris 1961, Sp. 551-552.

${ }^{8}$ HAYEZ, Conzié (wie Anm. 2), Sp. 212; Jean FAVIER, Les finances pontificales à l'époque du Grand Schisme d'occident 1378-1409, Paris 1966, S. 43-50. Entgegen den Behauptungen von Thomas FrENZ, Kämmerer (IV.), in: Lexikon des Mittelalters, Bd. 5, München 1991, Sp. 887-889, stand der Kämmerer nicht im Rang eines Kardinals - Conzié scheint mir das beste Gegenbeispiel zu sein. Allerdings hat man sich in der römischen Obödienz über diese Regel hinweggesetzt: Der Kämmerer der römischen Obödienz war Kardinal. 
überblickte alle Ausgaben. Als Inhaber dieses Amtes konnte er erwarten, einträgliche Pfründen zu erhalten. Fünf Jahre nach seiner Ernennung zum Kämmerer finden wir ihn als Erzbischof von Arles, 1390 wurde er Erzbischof von Toulouse und 1391 setzte ihn Clemens VII. an die Spitze der Erzdiözese von Narbonne9. Damit stand er der drittreichsten Diözese Frankreichs mit einem jährlichen Einkommen von etwa 27000 Gulden vor ${ }^{10}$. Mehrfach war François de Conzié Vikar von Avignon und 1411, nach dem Abzug der Truppen Benedikts XIII., wurde er dort Legat. Ein Jahr vor seinem Tod erhielt er schließlich durch Papst Martin V. den Titel des Patriarchen von Konstantinopel ${ }^{11}$.

\section{Conzié und die kuriale Politik 1381-1394}

Bereits 1379 hatte der Papst Conzié, damals noch Auditor, zusammen mit Graf Pierre von Genf nach Lausanne geschickt, um die Obödienz des dortigen Franziskanerstiftes sicherzustellen. Die zweite bekannte diplomatische Mission Conziés fand im Januar 1381 statt. Ausgestattet mit 1100 Gulden sollte er die beiden neutralen italienischen Kardinäle Brossano und Corsini von deren Konzilsplänen abbringen und sie stattdessen für die Avignoneser Obödienz gewinnen. Jeweils 500 Gulden waren für die Kardinäle bestimmt, das übrige Geld sollte der Bruder Corsinis, Abgesandter der Republik Florenz, erhalten ${ }^{12}$. Dieser ersten Mission nach Nizza folgte seine zweite Entsendung Mitte März erneut mit 1000 Gulden für die beiden Kardinäle ausgestattet, und eine dritte Reise im September ${ }^{13}$. Ein Jahr später (November 1382) wurde er als Nuntius in das Languedoc entsandt, um Geld und materielle Einnahmen der Kollektoren, wie Lebensmittel, an den Hof nach Avignon zu bringen ${ }^{14}$. Im Jahre 1384 mußte Conzié nach Marseille reisen, um Verhandlungen mit ortsansässigen Reedern zu führen. Papst Clemens VII. wollte Herzog Ludwig von Anjou bei dessen Eroberung des Königreichs Neapel unterstützen und dachte in diesem Zusammenhang an die Entsendung einer Flotte. Da niemand außer dem Papst und dem Kämmerer berechtigt war, Kredite im Namen der Kirche aufzunehmen, begab sich Conzié persönlich in die Hafenstadt. Abgesehen davon war der erfolgreiche Abschluß der Verhandlungen in Marseille von größter Wichtigkeit für die Machtkonsolidierung des Avignoneser Papstes auf der italieni-

\footnotetext{
${ }^{9}$ Konrad EubEL, Hierarchia Catholica Medii Aevi, Bd. 1, Münster 1898, S. 373, 515.

${ }^{10}$ BINZ, Népotisme (wie Anm. 2), S. 110.

${ }^{11}$ Ibid., S. 110 ; EUBEL, Hierarchia (wie Anm. 9), S. 287.

${ }^{12}$ Noël ValoIS, La France et le Grand Schisme d'Occident, 4 Bde., Paris 1896-1902, Bd. 2, S. 361; FAVIER, Finances (wie Anm. 8), S. 43.

${ }^{13}$ ValoIS, La France (wie Anm. 12), Bd. 2, S. 361.

${ }^{14}$ FAVIER, Finances (wie Anm. 8), S. 535. Es handelte sich um 3967 Dukaten. Ibid., S. 43.
} 
schen Halbinsel. Somit war die Auswahl der päpstlichen Delegation besonders brisant ${ }^{15}$.

Doch Conzié war primär Oberherr des Finanzwesens der päpstlichen Kurie. Das komplette Personal, vom Schatzmeister bis zum einfachen Schreiber der Kammer, war ihm unterstellt. Das Schisma führte an beiden päpstlichen Höfen zu einem erhöhten Geldbedarf. In Avignon mußte neben den ohnehin hohen Kosten eines päpstlichen Hofes zusätzlich ein ganzes Kollegium mit $36 \mathrm{Kar}-$ dinälen finanziert werden ${ }^{16}$. Daher war es dringend notwendig, daß Conzié die Arbeitsweise der Kammer straffer organisierte und neue Möglichkeiten fand, dringend benötigte Gelder zu beschaffen. Freilich konnte sein Dienstherr nur mit den Einkünften der Grafschaft Venaissin und den Abgaben aus seiner eigenen Obödienz rechnen. Deshalb stand in erster Linie Frankreich im Blickfeld finanzpolitischer Strenge. Clemens VII. erhöhte, wahrscheinlich nach Rücksprache mit Conzié, den finanziellen Druck auf den dortigen Klerus. Die geforderten Abgaben waren derart hoch, daß die Kirche Frankreichs völlig zu verarmen drohte ${ }^{17}$. Diese Finanzpolitik führte unweigerlich zu Spannungen zwischen der kurialen Verwaltung und dem französischen Klerus, der Rückhalt von der Pariser Universität erhielt ${ }^{18}$. Bereits beim Ausbruch des Schismas hatte sich die Universität zunächst dem königlichen Willen Karls V. widersetzt. Erst der unnachgiebige Kurs der Krone und vereinzelte Stimmen einiger Professoren brachte die Mehrheit der Dozenten in die Obödienz Avignons. Mit dem Tod König Karls V. im Herbst 1380 ließ der Druck auf die Universität anfänglich nach und die Rufe nach einem Kirchenkonzil mehrten sich nicht zuletzt deshalb, weil die päpstliche Finanzpolitik Anlaß zum Ärgernis bot ${ }^{19}$. In Herzog Ludwig von Anjou, Onkel und Regent für den noch minderjährigen König Karl VI., hatte die Kurie Avignons jedoch einen mächtigen Verbündeten am französischen Hof, der es schaffte, die Universität mundtot zu machen und alle Debatten, in denen es um eine Papstneuwahl, ein Generalkonzil oder ähnliches ging, zu verbieten ${ }^{20}$.

${ }^{15}$ FAVIER, Finances (wie Anm. 8), S. 51; Carl Joseph VON HEFELE, Conciliengeschichte, Bd. 6, 2, Freiburg i.Br. ${ }^{2} 1890$, S. 806.

${ }^{16}$ Bertrand SCHNERB, Les Armagnacs et les Bourguignons. La maudite guerre, Paris 1988, S. 30, HEFELE, Conciliengeschichte (wie Anm. 15), Bd. 6, S. 808.

${ }^{17}$ Chronica Karoli Sexti - Chronique du religieux de Saint-Denys, hg. von Louis BELLAGUET, Prosper Brugière Baron DE BARANTE, 6 Bde., Paris 1839-1852 (ND Paris 1994), Bd. 2, S. 2: Equidem Permissu Francie regis et procerum, velud libertatis et ecclesiarum regni vehemens impugnatur, earum patrimonia crebris decimis et usque ad supremam exinacionem statuit aterere.

${ }^{18}$ Chronique du religieux (wie Anm. 17), Bd. 2, S. 2.

${ }^{19}$ SCHNERB, Armagnacs (wie Anm. 16), S. 29.

${ }^{20}$ Chronique du religieux (wie Anm. 17), Bd. 2, S. 2: [...] nec de electione pape, nec de consilio generali faceret mencionem. 
Mit dem Tod des römischen Konkurrenten 1389 wurde Clemens VII. einziger Papst. Doch all seine Bemühungen, eine neue Papstwahl in Rom zu verhindern, scheiterten, und die Kardinäle Urbans VI. wählten 18 Tage nach dessen Tod Pietro Tomacelli (Bonifaz IX.) zu seinem Nachfolger, der nun versuchte, Frankreich in römische Obödienz zu ziehen. Abgesehen davon machte er Clemens das Angebot, sein erster Kardinal zu werden, wenn er abdanken würde ${ }^{21}$. Freilich dachte man in Avignon nicht im Traum an einen Amtsverzicht. Nicht nur Clemens VII., sondern sein kompletter Haushalt und das Kardinalskollegium mußten entschieden dagegen sein, wenn sie ihre Positionen behalten wollten. Vielmehr dachte man in Avignon an einen neuen Weg, den Papst in Rom auszuschalten, was mit der Schaffung eines neuen Königreiches (Adria) in Nord- und Ostitalien geschehen sollte. Im Süden Italiens lag das Reich von Neapel, dessen Herrscher seit der Rückeroberung durch Otto von Braunschweig Herzog Ludwig II. von Anjou war. Im November 1389 wurde letzterer durch Papst Clemens VII. in Avignon zum König gekrönt, was bedeutete, daß er sich in die Obödienz des Avignoneser Papstes begab. 1390 zog er nach Neapel, um die Stadt und gleichermaßen seinen Thron zu besetzen. Durch die Schaffung eines neuen Königreichs in Nordund Ostitalien wäre Clemens VII. am Ziel der Überlegungen für eine politische Zange gewesen ${ }^{22}$. Kandidat für diese neue Krone war der jüngere Bruder des Königs, Herzog Ludwig von Orléans, der nun zum leidenschaftlichen Verfechter der clementinischen Politik wurde.

Die Verhandlungen zogen sich in die Länge, weil der Papst ohne die Zustimmung des Kollegiums keine Länder der Kurie verkaufen oder verleihen konnte ${ }^{23}$. Auch Conzié erhob ernsthafte Bedenken gegen einen "Ausverkauf» des Kirchenstaates: Schließlich wollte der Papst seinen Sitz nach Italien verlegen. Ein Papst ohne Einkünfte aus eigenen Ländereien vor Ort wäre nicht oder kaum überlebensfähig gewesen ${ }^{24}$.

Anderthalb Jahre nach dem Beginn der Debatten über die Stiftung eines regnum Adriae, am 28. August 1394, lag die päpstliche Stiftungsurkunde am französischen Hof vor. Dennoch wurden die Verhandlungen abgebrochen. Diesmal ging die Unterbrechung von der französischen Seite aus, da zwei Punkte darin Grund zur Beanstandung gaben: Erstens sollte der Dukat Spoleto im Besitz des Papstes bleiben und zweitens verlangte Clemens VII., daß Ludwig von Orléans binnen sechs Monaten nach der Ausstellung der Urkunde einen militärischen Schlag gegen Papst Bonifaz IX. unternehmen solle. Herzog Ludwig von Orléans konnte den Hof in Paris jedoch unmöglich verlassen,

${ }^{21}$ HEFELE, Conciliengeschichte (wie Anm. 15), Bd. 6, S. $816 \mathrm{f}$.

${ }^{22}$ Christian DE MÉRINDOL, Ludwig I. von Anjou, in: Lexikon des Mittelalters, Bd. 5, München 1991, Sp. 2191f.; VAloIS, La France (wie Anm. 12), Bd. 2, S. 189, 194.

${ }^{23}$ Ibid., S. 194.

${ }^{24}$ Ibid. 
weil ihn sein kranker Bruder als Regenten für seinen Sohn einsetzte, falls seine Krankheit einen tödlichen Verlauf nehmen würde, und darauf deutete einiges hin $^{25}$. Drei Wochen nach dem Ausstellungsdatum der Urkunde für Herzog Ludwig von Orléans starb Papst Clemens VII. in Avignon.

Neun Tage nach dem Ableben Clemens' zogen sich die 21 Kardinäle ins Konklave zurück und wählten nach zwei Tagen Pedro de Luna (Benedikt XIII.) zum Nachfolger des verstorbenen Papstes. Die Papstwähler mußten vor ihrem Eintritt in das Konklave schwören, im Falle ihrer Wahl für die Einheit der Kirche zu kämpfen und, wenn nötig, auch zurückzutreten ${ }^{26}$. Aller Wahrscheinlichkeit nach wurde dieser Eid vor François de Conzié geleistet, da dieser in seiner Funktion als Kämmerer Hüter des Konklaves war und bereits unmittelbar nach dem Ableben des Papstes für den reibungslosen Ablauf sämtlicher Zeremonien zu sorgen hatte.

\section{Die Entwicklung unter Benedikt XIII.}

Obwohl die Könige von Frankreich und Aragón mit Sendschreiben eine Neuwahl verhindern oder wenigstens aufschieben wollten, haben wir gesehen, daß sich die Kardinäle in Avignon für eine Neuwahl entschieden. Den Boten des Königs von Frankreich hatte man zwar noch empfangen, aber der gewählte Dekan des Kardinalkollegs, Corsini, beschloß, das Schreiben vom Pariser Hof, dessen Inhalt er sich höchstwahrscheinlich denken konnte, erst nach dem Konklave zu öffnen ${ }^{27}$.

Zunächst deutete alles darauf hin, daß Benedikt XIII. tatsächlich das Ziel verfolgen würde, die Kircheneinheit wiederherzustellen. Er schwor abermals den bereits erwähnten Eid und lud das Kollegium zum Konsistorium. Doch der anfängliche Unionseifer Benedikts ermüdete sehr schnell, nachdem sich die Kardinalsversammlung auf keinen gemeinsamen Weg einigen konnte ${ }^{28}$.

Nun wuchs der Druck seitens der französischen Krone, die mehrere Abordnungen zu ihm schickte und Einsicht in den beschworenen Eid, die Wahlkapitulation, verlangte, was Benedikt jedes Mal verweigerte. Dieses verstockte Auftreten des Papstes und die Tatsache, daß bereits fast ein halbes Jahr ohne jegliches Handeln für die Kirchenunion verstrichen war, veranlaßte die Uni-

${ }^{25}$ Arnold ESCH, Bonifaz IX. und der Kirchenstaat, Tübingen 1969 (Bibliothek des Deutschen Historischen Instituts in Rom, 29), S. 141.

${ }^{26}$ Aldo LANDI, Il Papa deposto: Pisa 1409. L'Idea conciliare nel Grande Scisma, Turin 1985, S. 44.

${ }^{27}$ Martin Souchon, Die Papstwahlen zur Zeit des Großen Schismas, 2 Bde., Braunschweig 1898-1899 (ND in einem Bd., Aalen 1970), S. 210 f.

${ }^{28}$ Ibid., S. 242. 
versität von Paris, sich am 4. Februar 1395 öffentlich zum Cessionsweg zu bekennen ${ }^{29}$. Zur gleichen Zeit tagte ein französisches Nationalkonzil, das sich für die Abdankung beider Päpste aussprach ${ }^{30}$. Je mehr sich Benedikt gegen einen Rücktritt sträubte, desto mehr wuchs in weiten Teilen des französischen Klerus der Wunsch nach Verdrängung der päpstlichen Autorität. Die Universität von Paris stellte dem Papst nun ein regelrechtes Ultimatum: Würde Benedikt nicht bis zum 2. Februar 1398 zurücktreten, würde die Universität alles daran setzen, daß ihm die Obödienz entzogen werden würde ${ }^{31}$.

Die Universität von Paris hielt nach dem Verstreichen des Ultimatums Wort und unternahm tatsächlich alles für einen Obödienzentzug. Auf ihre Anregung hin versammelte der französische Hof eine Synode, die sich am 10. Juni 1398 mit einer Fünf-Sechstel-Mehrheit von Benedikt XIII. lossprach. Die Synodalen hatten sich vor der Abstimmung an die Krone gewandt und königlichen Schutz vor eventueller päpstlicher Rache erbeten ${ }^{32}$. François de Conzié hätte als Erzbischof von Narbonne ebenfalls am Konzil teilnehmen müssen, doch nur das Domkapitel von Narbonne sprach sich gegen Benedikt XIII. aus, ein Stimmzettel des Erzbischofs fehlt ${ }^{33}$.

Frankreich hatte den Papst von Avignon fallen lassen. Dieser konnte seine Beamten und sein Sicherheitspersonal ohne die französischen Einnahmen nicht mehr lange bezahlen, und es kam deshalb zu Unruhen in der Stadt.

Der Papst zog sich auf die Burg zurück und bereitete sich auf eine militärische Verteidigung vor ${ }^{34}$. Am 2. September 1398 verließen 18 der 27 Kardinäle und der größere Teil der kurialen Beamten Avignon und begaben sich nach Villeneuve, wo sie Truppen für ihren Schutz rekrutieren ließen. Zwei Wochen später entzogen sie ihrem Papst die Obödienz und begannen mit der Belagerung des Papstpalastes ${ }^{35}$.

Nachdem man sich innerhalb des Kardinalkollegiums nicht einig war, wie weiter vorzugehen wäre, und die Belagerung zu nichts führte, setzte man erneut auf Verhandlungen. Zu diesem Zweck erschien Anfang 1399 eine Gesandtschaft der Könige von Frankreich und Aragón in Avignon. Bedingt durch die langsam anwachsende Not in der belagerten Burg, versprach Benedikt XIII. abzudanken, wenn sein römischer Rivale das Gleiche täte, sterbe oder abgesetzt werden würde. Ferner erklärte er sich nun bereit, mit seiner Obödienz an einem Generalkonzil teilzunehmen, wenn dafür die Feindseligkeiten

\footnotetext{
${ }^{29}$ Ibid., S. 234ff.

${ }^{30}$ ValoIs, La France (wie Anm. 12), Bd. 3, S. $44 f f$.

${ }^{31}$ LANDI, Il papa (wie Anm. 26), S. 49.

${ }^{32}$ Ibid.

${ }^{33}$ Hélène MILLER, Emmanuelle Poulle, Le vote de la soustraction d'obédience en 1398, Bd. 1: Introduction, Édition et fac-similés des bulletins du vote, Paris 1988, S. 158.

${ }^{34}$ SouCHON, Papstwahlen (wie Anm. 27), S. 254.

${ }^{35}$ Ibid.
} 
eingestellt werden würden, wozu König Karl die Kardinäle anhalten sollte ${ }^{36}$. Die Gesandtschaft war erfolgreich. Benedikts Haushalt wurde auf 100 Personen reduziert und die Truppen des Papstes zogen aus der Burg ab. Der Papstpalast wurde mit einem hölzernen Palisadenzaun umgeben. Nur Lebensmittel durften zu Benedikt und seinem Gefolge durchgelassen werden ${ }^{37}$.

\section{Conzié und Benedikt XIII.}

War das Verhältnis zwischen ihm und Clemens VII. allem Anschein nach ausgezeichnet, muß die Beziehung zwischen Conzié und dem neuen Papst in Avignon von höchst komplizierter Natur gewesen sein. Tatsächlich kann nicht gesagt werden, in welchem Verhältnis die beiden zueinander standen. Doch auch wenn Favier davon ausgeht, daß Conzié sich dadurch auszeichnete, daß er mehr als nur einmal die Fronten wechselte, sich mal auf der Seite Benedikts, mal in den Reihen der unionsfreudigen Kardinäle befand, konnte ich ein solches Verhalten des Kämmerers nicht erkennen: Natürlich weiß man, daß Conzié Unterstützer der Konzilien von Pisa und Konstanz war und deshalb seinen Dienst bei Benedikt XIII. quittierte. Auch ist bekannt, daß er als Gläubiger König Sigismunds in Erscheinung trat, letzteren während der Verhandlungen von Narbonne und Perpignan bewirtete und sich in diesem Zusammenhang energisch gegen Pedro de Luna aussprach. Doch wie er zum neu gewählten Benedikt XIII. von 1394 stand, kann bis heute nicht vollständig geklärt, sondern nur erahnt werden. Zunächst muß gesagt werden, daß Clemens VII. eine hochverschuldete Kurie hinterließ. Diesen Zustand finanzieller Not zu beheben, mußte vordringlichstes Anliegen des neuen Papstes sein, der zu jeder Zeit größten Wert auf finanzielle Sicherheit legte. Der Kämmerer war als Oberherr der päpstlichen Finanzverwaltung zu einem gewissen Grad mitverantwortlich für die leeren Kassen, die er nun, im Dienste Benedikts, wieder zu fuullen hatte. Möglicherweise hatte es in der Vergangenheit, während des Pontifikats Clemens VII., Gründe gegeben, die eine Distanzierung zwischen Conzié und dem späteren Papst nach sich zogen. Sowohl Kardinal Luna als auch Conzié hatten zunächst hohes Ansehen bei Papst Clemens VII. genossen. Beide wurden mit wichtigen Missionen betraut und beide enttäuschten ihren Papst nicht, sondern kehrten jedes Mal erfolgreich nach Avignon zurück. Conzié gelang es, die römischen Kardinäle Brossano und Corsini für Clemens zu gewinnen und Luna konnte die spanischen Königreiche in Avignoneser Obö-

${ }^{36}$ Ibid., S. 259.

${ }^{37}$ Ibid., S. 260. 
dienz führen ${ }^{38}$. Es lag sicherlich nicht an Kardinal de Luna, wenn die Unterredungen mit den englischen Gesandten scheiterten. Doch sein Verhältnis mit dem Papst mußte sich daraufhin unweigerlich abkühlen ${ }^{39}$. Aber nicht nur die gescheiterten Missionen des Kardinals in Lelling und Paris waren dafür verantwortlich, wenn man bedenkt, daß er sich immer öfter in Gegenwart Clemens für die Union der Kirche aussprach (ganz gleich wie er später als Papst $z u$ diesem Thema stehen würde), was den Papst unweigerlich in Verlegenheit brachte $^{40}$. Conzié konnte sich allem Anschein nach eine Kircheneinheit nur unter den Päpsten von Avignon vorstellen, da andernfalls sein eigener Posten als Kämmerer verloren gewesen wäre. Die gute Beziehung zwischen Kämmerer und seinem Vorgänger konnte Papst Benedikt XIII. aus einem weiteren Grund verärgern, da er Clemens VII. als Geldverschwender erachtete. Conzié konnte in den Augen Benedikts deshalb leicht das übergebliebene Relikt einer unbedacht ausschweifenden Finanzpolitik werden.

Gibt es mehrere Hinweise für ein schlechtes Verhältnis zwischen Conzié und dem Nachfolger Clemens VII., so gilt es, an dieser Stelle auch Faktoren zu nennen, die von einer guten Zusammenarbeit von Kämmerer und Benedikt XIII. zeugen. In diesem Kontext sollte man besonders die Haltung Conziés im Verlauf des ersten Obödienzentzugs von 1398 betrachten. Als sich die nationale Kirchensynode unter Simon de Cramaud für eine Loslösung von Benedikt aussprach, stimmte er in seiner Funktion als Erzbischof von Narbonne, wie wir bereits gesehen haben, nicht ab. Er war nämlich nicht anwesend, und auch wenn das Domkapitel seiner Bischofsstadt für den Entzug war, fehlt jeder Hinweis darauf, daß damit auch der Wille des Erzbischofs ausgedrückt wurde ${ }^{41}$. Es ist unwahrscheinlich, daß ausgerechnet Conziés Stimmzettel im Laufe der Jahrhunderte verloren ging und deshalb nicht überliefert ist. Vielmehr könnte diese Enthaltung darauf hinweisen, daß Conzié den päpstlichen Stuhl von Avignon erhalten wollte, da er somit das Amt des Kämmerers weiter ausüben konnte.

\section{Der Obödienzentzug von 1398}

Tatsächlich ist es so, daß man Conzié nach der erfolgten Loslösung Frankreichs von Benedikt keiner Seite eindeutig zuzuordnen vermag. Zwar war Conzié kein Franzose von Geburt, durch sein Erzbischofsamt in Narbonne

\footnotetext{
${ }^{38}$ FAVIER, Finances (wie Anm. 8), S. 43.

${ }^{39}$ Chronique du religieux (wie Anm. 17), lib. XIV, S. 3.

${ }^{40}$ SOUCHON, Papstwahlen (wie Anm. 27), S. 224.

${ }^{41}$ MILLER, POULLE, Le vote (wie Anm. 33), S. 158.
} 
aber dennoch Untertan des französischen Königs. Es fehlen klare Angaben für Conziés räumlichen Aufenthalt während Benedikts Belagerung durch die Kardinäle. Er mag sich bei Benedikt in der Burg, beim Kollegium in Villeneuve oder an einem ganz anderen Ort aufgehalten haben ${ }^{42}$.

Nach dem Ausgleich durch die Vermittlung Frankreichs und Kastiliens ernannte der französische Hof François de Conzié zum Hüter des Papstes in dessen eingeschlossenem Palast ${ }^{43}$. Gerade diese Tatsache entpuppt sich bei genaueren Untersuchungen als sehr ambivalente Episode. Unter dem Aspekt, daß er bei der nationalen Kirchenversammlung am 10. Juni 1398 nicht abgestimmt hatte, erhält seine Rolle nach der Belagerung und den Einigungsgesprächen eine sehr interessante Bedeutung. Wozu benötigte man überhaupt einen Hüter des Papstes? Zunächst ist es nicht von der Hand zu weisen, daß dies juristische Ursachen hatte. Um nicht diplomatische Verstimmungen bei anderen Fürsten Europas hervorzurufen, durfte nicht der Eindruck entstehen, daß der Papst ein Häftling war. Abgesehen davon war Avignon kein französisches Territorium und die Regierung Karls VI. hatte sicherzustellen, daß nicht die Vermutung aufkäme, sie bemächtige sich päpstlichen Landes. Darüber hinaus bekannte sich Frankreich mit dem Obödienzentzug zur Neutralität und nicht zum Gegenpapst in Rom. Abgesehen davon war Benedikt immer noch Papst. Er wurde weder abgesetzt, noch von allen »seinen « Fürsten verlassen (Schottland und Aragón). Dem Papst durfte unter keinen Umständen etwas zustoßen, und deshalb benötigte man einen kompetenten Mann. Der Kämmerer war aus mehreren Gründen der ideale Kandidat für den Posten eines »Papsthüters«. Zum einen war er »neutral«, hatte er doch öffentlich beim Obödienzentzug weder für, noch gegen Benedikt XIII. gestimmt. Zum anderen deckte sich sein zusätzliches Aufgabengebiet in einigen Punkten mit dem des Kämmerers (wenn man bedenkt, daß er Lebensmittel beschaffen und verteilen mußte) $)^{44}$. Doch waren es nicht nur diese Überlegungen, die die französische Regierung zum Entschluß brachten, den apostolischen Kämmerer als Garanten für das Wohlergehen des Papstes zu ernennen. Vielmehr sollte man den Einfluß der Herzöge im Regierungsrat, insbesondere den Ludwigs von Orléans, nicht vergessen. Dieser war nämlich an der Absetzung Benedikts XIII. nicht interessiert, konnte er doch nur mit ihm weiter auf sein eigenes Königreich hoffen. Gerade unter dem Eindruck des Fernbleibens Conziés von der Nationalsynode 1398 erscheint es als sehr wahrscheinlich, daß er, wie Herzog Ludwig von Orléans, allem Anschein nach nicht für das Fallenlassen Benedikts war. Ganz im Gegenteil: Ich vermute eine Zusammenarbeit von Kämmerer und Herzog, weil Conzié gegen den Obödienzentzug war und nur mit dem Erhalt des Papstes von Avignon seine eigene Stellung gesichert wußte.

42 SoUCHON, Papstwahlen (wie Anm. 27), S. 250.

${ }^{43}$ HAYEZ, Conzié (wie Anm. 2), Sp. 213.

${ }^{44}$ SOUCHON, Papstwahlen (wie Anm. 27), S. 260. 
Günstig für die Ziele des Kämmerers und Ludwigs von Orléans waren die Entwicklungen im Lager der Kardinäle Benedikts. In der Zwischenzeit waren diese in zwei Fraktionen gespalten, wobei die Mehrheit im Kollegium eine Versöhnung mit dem Papst anstrebte ${ }^{45}$. Die gewünschte Verständigung erfolgte sicherlich vor der Überlegung, da $\beta$ ein gefangengenommener Pontifex nichts für die Kircheneinheit unternehmen könne. Langsam bahnte sich eine deutliche Tendenz an, die Obödienz des Papstes wiederherzustellen. Einer, der dafür alle nur erdenklichen Hebel in Bewegung setzte, war ganz offensichtlich der Herzog von Orléans. Wieso sonst beschwerte sich im September 1401 ausgerechnet die Universität von Orléans beim König über den erfolgten Obödienzentzug? ${ }^{46}$ Auch Conzié dürfte sich für Benedikt eingesetzt haben. Man weiß von einem regen geheimen Nachrichtenaustausch zwischen Papstpalast und Aragòn; dieser wird Conzié, der als Kämmerer mit den internen Strukturen am päpstlichen Hof bestens vertraut war, schwerlich verborgen geblieben sein.

Der Papst scheint auf den ersten Blick 1399 nicht erfreut über die Wahl seines Hüters gewesen zu sein, da er 1401 vom König einen anderen wünschte. Der König erfüllte diesen Wunsch und ernannte den Herzog Ludwig von Orléans selbst ${ }^{47}$. Bedenkt man, daß es Benedikt daraufhin gelang, aus dem Palast zu fliehen, woran Orléans allem Anschein nach beteiligt war, wird man annehmen dürfen, daß Conzié eine solche Flucht nicht zugelassen hätte. Andererseits ist zu bedenken, daß der Herzog von Orléans einen weit größeren weltlichen Einfluß besa $\beta$ und erheblich mehr Beziehungen spielen lassen konnte als Conzié. Vielleicht handelte Benedikt demnach sogar mit Conziés Unterstützung oder auf dessen Ratschlag, als er König Karl VI. darum bat, Ludwig von Orléans als Wächter für ihn zu bestimmen. In diesem Fall müßte man annehmen, daß Conzié mit dem Bruder des Königs in Kontakt stand.

Die Reaktionen aus dem Lager der Kardinäle auf die Flucht des Papstes legen die Vermutung nahe, daß nicht nur Orléans hinter dem Entkommen Benedikts stand. Im Gegenteil deutet alles darauf hin, daß eine Verbindung zwischen dem Herzog von Orléans und dem Kardinalskollegium bestand. Möglicherweise war Conzié gar der Mittelsmann.

Kurze Zeit nach dem Protestschreiben der Universität von Orléans erreichte eine ähnliche Schrift der Universität Toulouse den französischen Hof, was unverzüglich heftige Reaktionen der Universität von Paris hervorrief ${ }^{48}$. Verantwortlich für die Toulouser Thesen könnten der Kämmerer oder die aragonesische Krone gewesen sein, da der Hof in Avignon, wie bereits erwähnt,

${ }^{45}$ Ibid., S. 257.

${ }^{46}$ LANDI, Il papa (wie Anm. 26), S. 58. Es könnte auch Conzié gewesen sein, der seine ehemalige Universität dazu anhielt.

${ }^{47}$ SoucHON, Papstwahlen (wie Anm. 27), S. 263.

${ }^{48}$ Ibid., S. 264. 
nicht von der Außenwelt abgeschnitten war und durchaus verschiedene Aufträge an unterschiedliche Instanzen erteilt haben könnte. Sicherlich spielte die große Rivalität zwischen den beiden Universitäten von Paris und Toulouse eine nicht zu unterschätzende Rolle, wenn letztere eine Verteidigung Benedikts publizierte. Um jedoch der anderen Seite auch gerecht zu werden, muß selbstverständlich gesagt werden, daß alles vollkommen anders gewesen sein könnte. Ein Entkommen Benedikts wäre unter Conzié niemals möglich gewesen, weil dieser zu streng über den Papst wachte und es reiner Zufall war, daß die Universitäten von Orléans und Toulouse zu dem Entschluß kamen, die Obödienz Benedikts XIII. wiederherzustellen. Es bliebe dann allerdings fragwürdig, warum Conzié beim Obödienzentzug von 1398 nicht abstimmte und sich somit eindeutig für eine Seite entschied.

\section{Von Avignon nach Pisa}

In der Nacht auf den 12. März 1403 floh Papst Benedikt XIII. aus seinem Palast und zog sich nach Château-Renard zurück, wo er sich mit seinen Kardinälen aussöhnte ${ }^{49}$. Einen Monat später stellte der König von Kastilien und am 28. Mai 1403 Karl VI. die Obödienz Benedikts XIII. wieder her ${ }^{50}$. Conzié befand sich aller Wahrscheinlichkeit nach beim Papst, der in der Zwischenzeit nach Marseille übergesiedelt war und sich dort im Kloster Saint-Victor niederließ, wo er im Oktober den Herzog von Orléans empfing ${ }^{51}$. Immer davon ausgehend, daß Conzié an der Kurie weilte, zog er im Mai 1404 zusammen mit Benedikt XIII. nach Genua. Inwieweit Conzié dafür verantwortlich war, daß der Papst von Genua aus eine Gesandtschaft nach Rom zu Bonifaz IX. schickte, um diesem den Rücktritt nahezulegen, muß ungeklärt bleiben. Allerdings sollte man bedenken, daß der Kämmerer allem Anschein nach zum damaligen Zeitpunkt von der Rechtmäßigkeit seines Papstes überzeugt war und deshalb Interesse gehabt haben könnte, Bonifaz IX. ausgeschaltet zu wissen ${ }^{52}$. Nachdem im Spätsommer 1405 eine Pestepidemie in Genua ausbrach, verließen Conzié, der Papst und die Kurie die Stadt und zogen sich langsam in Richtung Savona zurück, wo sie im Oktober 1405 ankamen ${ }^{53}$.

${ }^{49}$ LANDI, Il papa (wie Anm. 26), S. 59. Übrigens hatte zu diesem Zeitpunkt Robert de Braquemont, eine Kreatur des Herzogs von Orléans, den Wachdienst.

${ }^{50}$ HeFELE, Conciliengeschichte (wie Anm. 15), Bd. 6, S. 871, 874.

${ }^{51}$ Le Diare de François de Conzié, hg. von Marc DYKMaNs, in: DERs. (Hg.), Le Cérémonial Papal de la fin du Moyen Âge à la Renaissance, Bd. 3, Rom, Brüssel 1983 (Bibliothèque de l'Institut historique belge de Rome, 26), S. 93.

${ }_{52}^{52}$ LANDI, Il papa (wie Anm. 26), S. 65.

${ }^{53}$ Le Diare de François de Conzié (wie Anm. 51), S. 93. 
Am 26. Juni 1406 brach die Kurie in Richtung Marseille auf, weil die Pestepidemie aus Genua inzwischen Savona erreicht hatte ${ }^{54}$. Am 28. Juni weilte der Papst im Castello Gavone der markgräflichen Familie del Carretto. Drei Tage später war Conzié anwesend, als eine kastilische Delegation den Papst erneut um seinen Rücktritt bat, was letzterer jedoch abermals ablehnte ${ }^{55}$. Während Benedikt XIII. am 19. Juli Gavone mit dem Schiff verließ, um vor der sich ausbreitenden Pest nach Monaco und von dort aus weiter nach Nizza zu fliehen, reiste sein Kämmerer auf dem Landweg voraus, um Benedikts Einzug in Nizza vorbereiten zu können. Conzié blieb deshalb der Kurie in der Zeit zwischen 19. Juli und 17. August $1406 \mathrm{fern}^{56}$. Nachdem er der Stadt die nahende Ankunft des Papstes gemeldet hatte, zog er es allem Anschein nach vor, Nizza zu verlassen und sich ins Hinterland zurückzuziehen. Dieses Verhalten muß im Zusammenhang mit der Pestepidemie gesehen werden, da Conzié zu den Mitgliedern der Kurie gehörte, die bereits in Finale Benedikt drängten, den Ort zu verlassen ${ }^{57}$. In der Zwischenzeit hatte der Papst eine neue Abgabe seiner Obödienz gefordert, womit seine Reisen finanziert werden sollten ${ }^{58}$. Es ist durchaus anzunehmen, daß er seinen Kämmerer schon früher in Monaco damit betraut hatte, diese Steuer in Frankreich einzufordern.

Drei Monate später, am 5. November 1406, verstarb Innozenz VII. in Rom. Am 13. November 1406 informierte man Conzié in Fréjus vom Tod des römischen Papstes und der Kämmerer beschloß, Benedikt erst in Toulon (20. oder 21. November) davon zu berichten ${ }^{59}$. Souchon erwähnt, daß Benedikt XIII. sich eine zweiwöchige Bedenkfrist erbeten habe, nachdem er vom Ableben seines Gegners erfahren hatte ${ }^{60}$. Tatsächlich war es aber so, daß Conzié die Neuigkeit bewußt zurückhielt. Innozenz VII. verstarb bereits am 5 . November. Am 13. November gelangte die Nachricht zu Conzié, und erst eine Woche später gab er die Information an den Papst weiter. Es ist nicht von der Hand zu weisen, daß diese Episode die Theorie, Conzié sei nicht an der Absetzung seines Papstes interessiert gewesen, untermauert. Benedikt XIII. gewann damit zusätzliche Zeit, denn als er vom Tod Innozenz VII. erfuhr und damit eigentlich zum Rücktritt gezwungen wurde, war in Rom bereits ein Nachfolger (Gregor XII.) gewählt. Eine Tatsache, die Conzié durchaus bekannt gewesen sein konnte ${ }^{61}$. Ganz gleich, ob man in Frankreich wußte, daß Conzié für die Verzögerung verantwortlich war oder nicht: Der Druck auf Benedikt und seine Kurie seitens der unionsfreudigen Kardinäle und der Regierung Karls VI. er-

\footnotetext{
${ }^{54}$ Le Diare de François de Conzié (wie Anm. 51), S. 372.

${ }^{55}$ Ibid., S. 376.

${ }^{56}$ Ibid., S. 96.

${ }^{57}$ Ibid., S. 97. Schließlich verstarb ein Mitglied des Kollegiums an der Pest.

${ }^{58}$ HEFELE, Conciliengeschichte (wie Anm. 15), Bd. 6, S. 878.

${ }^{59}$ Le Diare de François de Conzié (wie Anm. 51), S. 387 f.

${ }^{60}$ Souchon, Papstwahlen (wie Anm. 27), S. 267.

${ }^{61}$ Ibid., S. 257.
} 
höhte sich. Ein neues Nationalkonzil des französischen Klerus (wieder ohne den Erzbischof von Narbonne) beschloß am 4. Januar 1407, daß es dem Papst untersagt sei, Gelder der französischen Kirche zu fordern. Doch war die Tatsache, daß die Kirchenversammlung erneut festlegen ließ, daß nur eine allgemeine Kirchenversammlung beider Obödienzen das Schisma beenden könne, wesentlich gefährlicher für Benedikt ${ }^{62}$. Die Überlegungen der französischen Synode dehnten sich auch auf Gregor XII. aus, so daß der römische Papst Briefe an Benedikt XIII. schreiben ließ, indem er ihm erste Schritte unterbreitete, um ein Treffen zu arrangieren und die Cessio beider Kirchenoberhäupter $\mathrm{zu}$ vollziehen ${ }^{63}$. Unter dem Eindruck des französischen Kirchenkonzils schickte Benedikt am 31. Januar 1407 von Marseille aus ein Schreiben an Gregor, in welchem er der römischen Forderung nachkam ${ }^{64}$. Wenig später wurde Savona als Treffpunkt ausgewählt, wobei die Wahl des Ortes Bedenken in Frankreich aufkommen ließ, da Savona äußerst ungünstig für Gregor war. Benedikt rechnete damit, daß sein römischer Rivale nicht erscheinen würde, und tatsächlich erhob Gregor Einwände und bat um eine Verlegung, was Benedikt, wie zu erwarten war, strikt ablehnte und stattdessen eifrig mit den Reisevorbereitungen für seinen Aufbruch nach Savona begann ${ }^{65}$. Die Angst vor einem Nichtzustandekommen des Treffens breitete sich unter den Mitgliedern beider Kollegien aus. In Savona angekommen, fand Benedikt natürlich keinen römischen Papst vor. Nur die eindringlichsten Überredungskünste der Kardinäle Avignons konnten Benedikt dazu bewegen, Pisa als alternativen Treffpunkt mit Gregor zu akzeptieren ${ }^{66}$. Dort wurde Benedikt im September 1407 von einer Gesandtschaft Gregors aufgesucht, die ihm Porto Venere oder Pietrasanta als Tagungsort vorschlugen. Nach längerer Überzeugungsarbeit ließ sich der Papst von Avignon darauf ein, akzeptierte Porto Venere und ging bei La Spezia vor Anker, während Gregor 75 Kilometer entfernt in Lucca Stellung bezog. In der Zwischenzeit hatte es Spannungen zwischen Gregor XII. und seinem römischen Kardinalskollegium gegeben. Wie im Falle Benedikts XIII. von 1398 warfen die Kardinäle ihrem Papst vor, er kümmere sich nicht um die Union der Kirche und nähme ihnen immer mehr Rechte im Konsistorium. Dieser Konflikt rief Benedikt auf den Plan, der das Geschehen in Lucca aufmerksam verfolgte. Die Loslösung des römischen Kardinalats von ihrem Papst würde eine nahezu tödliche Gefahr für den Herrschaftsanspruch Gregors bedeuten, was Benedikt zu seinen Gunsten ausnützen konnte und wollte.

Die Anfeindungen zwischen Gregor und seinen Kardinälen führten dazu, daß die meisten Mitglieder des römischen Kollegiums in der Nacht vom 11.

${ }^{62}$ Howard KAMINSKY, Simon de Cramaud and the Great Schism, New Jersey 1983, S. 268.

${ }^{63}$ SOUCHON, Papstwahlen (wie Anm. 27), S. 270.

${ }^{64}$ Ibid.

${ }^{65}$ HefELE, Conciliengeschichte (wie Anm. 15), Bd. 6, S. 897.

${ }^{66}$ SOUCHON, Papstwahlen (wie Anm. 27), S. 273. 
auf den 12. Mai 1408 ihren Herrn verließen und sich nach Pisa begaben. Dort angekommen, wandten sie sich sofort mit einem Schreiben an Benedikt und dessen Kollegium und forderten sie auf, sich mit ihnen zu vereinen und ein gemeinsames Treffen in Livorno abzuhalten ${ }^{67}$. Benedikt ließ sich auf ein Treffen ein, wollte jedoch nicht persönlich anwesend sein und schickte vier Kardinäle und drei Erzbischöfe zum Kollegium Gregors nach Livorno. Bereits am 20. Mai trafen diese bei ihren römischen Kollegen in der Hafenstadt ein ${ }^{68}$.

Vor dem Hintergrund der Zusammenkunft beider Kardinalskollegien in Livorno bahnte sich die Loslösung Conziés von seinem Papst an. Als Papst Gregor XII. von seinen Kardinälen verlassen wurde und Benedikt XIII. die genannte Abordnung zu diesen nach Livorno schickte, konnte er nicht wissen, $\mathrm{daß}$ sich seine Kardinäle denen des römischen Gegners anschließen würden. Zunächst blieb Conzié bei seinem Papst und reiste mit diesem nach Spanien $a b$, nachdem er zusehen mußte, daß mehr und mehr Kardinäle den Anschluß an das Kollegium beider Obödienzen in Livorno suchten. Wann sich der Abfall Conziés vollzog, kann nicht genau gesagt werden. Irgendwann zwischen Mai und Oktober 1408 löste sich der Kämmerer von Benedikt und wechselte in das Lager des pisaner Konzils. Die Gründe für dieses Verhalten Conziés sind vielseitig. Zum einen ergingen Ende Juni 1408 Schreiben an die zwei Kurien, in welchen das zusammengeführte Kollegium die Prälaten der jeweiligen Obödienzen zu einer Synode aufriefen. Man kann davon ausgehen, daß dieses Schreiben spätestens nach drei Wochen, also Mitte Juli, bei Benedikt vorlag. Die darin erwähnte Synode war für Lichtmeß 1409 angesetzt ${ }^{69}$. Am 14. Juli 1408 erfolgte die offizielle Ausschreibung des Konzils. Es wurde für den 25. März (Mariä Verkündigung) 1409 angesetzt und Tagungsort sollte die Stadt Pisa sein. Zur gleichen Zeit erfolgte ein erneutes Mahnschreiben an die Prälaten der beiden Obödienzen, das spätestens nach einem Monat auch bei Benedikt, der inzwischen in Perpignan weilte, eintraf ${ }^{70}$. Nachdem die Kardinäle Gregors am 11. Oktober sämtliche Prälaten, Beamten, Bediensteten und alle noch an dessen Kurie befindlichen Personen aufforderten, ihn zu verlassen, liegt es nahe, daß ein ähnliches Schreiben auch an die Kurie Benedikts erging $^{71}$. Dies würde sich auch mit der Tatsache decken, daß dieser seit Oktober 1408 einen kanonischen Prozeß gegen Conzié anstrebte ${ }^{72}$. Tatsächlich schickten die Kardinäle Benedikts am 24. September 1408 ein Schreiben, das am 22. Oktober 1408 ankam. Dennoch halte ich es für sehr wahrscheinlich,

${ }^{67}$ Ibid., S. 274.

${ }^{68}$ Ibid., S. 275.

${ }^{69}$ HefELE, Conciliengeschichte (wie Anm. 15), Bd. 6, S. 913

${ }^{70}$ Giovanni D. MANSI, Sacrorum Conciliorum nova et amplissima collectio, Bd. 26, Florenz, Venedig 1784, S. 1131, 1161.

${ }^{71}$ HeFELE, Conciliengeschichte (wie Anm. 15), Bd. 6, S. $917 \mathrm{f}$.

${ }^{72}$ HAYEZ, Conzié (wie Anm. 2), S. 213. 
daß sich Conzié schon zuvor von Benedikt losgesagt hatte. Grund dafür liefert eine ganz einfache Überlegung: Conzié war kein Schismatiker und er wollte die Kircheneinheit. Man muß nur davon ausgehen, daß die Nationalsynode von 1398 nach Auffassung Conziés nicht dazu berechtigt war, einem rechtmäBig gewählten Papst die Obödienz zu entziehen. Nachdem nun die Kollegien beider Päpste in Livorno vereint waren und gemeinsam ihre Herren zum Rücktritt bewegen wollten, war in den Augen Conziés eine Autorität geschaffen, der sowohl Papst Gregor XII. als auch Benedikt XIII. gehorchen mußten. Jetzt stellte sich heraus, daß die beiden Oberhirten nicht daran dachten abzudanken $^{73}$. Conzié mußte feststellen, daß sich sein Dienstherr als Schismatiker entpuppte, weil er der Ladung nach Pisa nicht nachkam und das Berufungsrecht der Kardinäle mißachtete ${ }^{74}$. Doch neben diesen kirchenrechtlich religiös motivierten Gründen war es sicherlich auch ein gewisser Pragmatismus, der den Kämmerer dazu veranlaßte, seinem Papst den Rücken zu kehren. Die Position Benedikts war mehr und mehr ausweglos geworden und eine Unterstützung des Papstes seitens Conziés konnte zur persönlichen Gefahr für ihn werden. Darüber hinaus war Benedikt seiner wichtigsten Stütze im französischen Kronrat beraubt worden: Bereits am 23. November 1407 hatte Herzog Philipp von Burgund seinen Neffen Ludwig von Orléans ermorden lassen. Mit dem Wegfall des Herzogs verringerte sich der Druck auf die Universität und die Diskussion über einen erneuten Obödienzentzug wurde lauter. Am 12. Januar 1408 drohte Karl VI. mit der Subtraktion, worauf Benedikt seinerseits eine Bulle verfaßte, nach der der Obödienzentzug mit Exkommunikation bestraft werden würde ${ }^{75}$. Würde Conzié weiterhin bei Benedikt bleiben und letzterer den Machtkampf mit Frankreich und den Kardinälen beider Obödienzen verlieren (was immer wahrscheinlicher wurde), würde er leicht sowohl seinen Bischofssitz, als auch sein kuriales Amt verlieren (ohne Kurie kein Kämmerer). Würde er sich auf die Seite des zusammengeführten Kollegiums stellen, das nach Auffassung Conziés ohnehin dem Papst übergeordnet war, könnte er immerhin seinen Bischofssitz in Narbonne retten und hätte gute Aussichten auf den Erhalt seines Amtes.

Am 4. Januar 1409 finden wir Conzié in Villeneuve-lez-Avignon. Die ehemaligen Kardinäle Benedikts waren zum Teil dorthin zurückgekehrt, um endgültige Vorbereitungen für das Konzil in Pisa zu treffen. Der abtrünnige Kämmerer erscheint hier als kompetenter Informant, der ihnen alles erzählen konnte, was sich in der Zwischenzeit bei Benedikt in Perpignan abgespielt hatte $^{76}$.

\footnotetext{
${ }^{73}$ SoUCHON, Papstwahlen (wie Anm. 27), S. 284f.

${ }^{74}$ Remigius BÄUMER, Die Entwicklung des Konziliarismus. Werden und Nachwirken der konziliaren Idee, Darmstadt 1976, S. 1431.

${ }^{75}$ HEFELE, Conciliengeschichte (wie Anm. 15), Bd. 6, S. 908

${ }^{76}$ Le Diare de François de Conzié (wie Anm. 51), S. 108.
} 
Kurze Zeit vor der geplanten Eröffnung des Konzils drang der unter römischer Obödienz stehende König Ladislaus von Neapel in florentinisches Gebiet ein, um die Kardinäle an ihrer Versammlung zu hindern. Der König konnte jedoch zurückgedrängt werden und das Konzil begann termingerecht am 25. März $1409^{77}$.

Auf der fünften Sitzung (24. April) wurde eine Denkschrift zur Entstehung und zum Fortgang des Schismas verfaßt. Sie sollte die Einleitung zum Prozeß gegen die beiden Prätendenten $\operatorname{sein}^{78}$. Der eigentliche Prozeßauftakt fand erst nach langen Zeugenbefragungen durch eine eigens dafür gegründete Kommission auf der neunten Sitzung statt. Am 5. Juni 1409 fällte die Synode das Urteil und ließ es durch den Patriarchen Simon de Cramaud bei offener Türe des Doms verlesen.

Pedro de Luna und Angelo Correr, die früher Benedikt XIII. und Gregor XII. genannt wurden, erklärte man für notorische Schismatiker und Nährer des notorischen und schrecklichen Verbrechen des Meineides. Beide haben sich, so das Urteil, aller Ehren und Ämter unwürdig gemacht, und die Synode sprach die definitive Sentenz der Beraubung, Absetzung und Ausschließung über sie und verbot ihnen, sich als Papst zu benehmen ${ }^{79}$. Es wurde ferner festgestellt, daß die römische Kirche nun vakant sei, womit das Konzil alle Gläubigen, auch Kaiser und Könige, von jedem Eid und jeder Verpflichtung gegen die beiden Genannten entband. Das Konzil verbot jedem Christen, einem oder beiden Prätendenten in irgendeiner Weise zu gehorchen oder mit Rat und Tat beizustehen.

Besonders die Tatsache, daß das Konzil die Sedisvakanz des Heiligen Stuhles konstatierte, rief Conzié ins Zentrum der nun folgenden Geschehnisse, denn die Synode mußte einen neuen Papst wählen. Es ist anzunehmen, daß Conzié mit seinem ehemals römischen Amtskollegen, Kämmerer Caraccioli, gemeinsam die Planung und die Vorbereitung des Konklaves übernahm ${ }^{80}$. Da Caraccioli als Kardinal Mitglied im Konklave war und bereits im Vorfeld der Wahl verschiedene Besprechungen in seiner Funktion als Papstwähler zu führen hatte, ist anzunehmen, daß Conzié die meisten Vorbereitungen selbst übernommen hat. Als erstes mußte der passende Raum gefunden werden, der das angereiste Kardinalskolleg fassen konnte. Die einzige Möglichkeit, das Konklave abzuhalten, bot der erzbischöfliche Palast der Stadt Pisa. Es handelte sich dabei um einen beträchtlichen Gebäudekomplex, der ein rechteckiges

\footnotetext{
${ }^{77}$ HEFELE, Conciliengeschichte (wie Anm. 15), Bd. 6, S. 936.

${ }^{78}$ Ibid., S. 1006.

${ }^{79}$ Thesaurus Novus Anectotorum, hg. von Edmond MARTĖNE, Ursin DURAND, Bd. 2, Paris 1717 (ND Paris 1968), S. 1478: et nihilominus ipsos Petrum et Angelum et eorum utrumqueper hanc sententiam definitivam in his scriptis privat, abjicit et praescindit; inhibendo eisdem, ne eorum aliquis pro summo Pontifice gerere se praesumat [...].

${ }^{80}$ SOUCHON, Papstwahlen (wie Anm. 27), S. 54f., $278 f$.
} 
Atrium umschloß und an zwei Seiten von einem alten Garten umgeben war. Kurze Zeit nachdem man die Wahl beschlossen hatte, begann man auf Anweisung Conziés und Caracciolis die Fenster des Gebäudes, welche auf die Straße blickten, zuzumauer ${ }^{81}$. Damit die Kardinäle keinen Überfall der abgesetzten Päpste, insbesondere Gregors, fürchten mußten, sorgten Conzié und sein römischer Kollege auch für die Sicherheit, indem sie auf den Stadtmauern Soldaten postieren ließen. Die Mannschaft der pisanischen Zitadelle wurde auf 500 Mann aufgestockt.

Am 15. Juni 1409 versammelten sich alle Mitglieder des Konzils in der Kathedrale, um ein Hochamt zu feiern, das durch den Erzbischof von Lyon, Philipp de Thury, abgehalten wurde. Am Abend schließlich, zwischen 20 und $21 \mathrm{Uhr}$, fand der feierliche Einzug der 23 Kardinäle in das Konklave statt ${ }^{82}$. Bereits im Vorfeld wurden vier Herren zu den Hütern des Konklaves ernannt. Ihr Vorsteher dürfte François de Conzié selbst gewesen sein. Dafür spricht, wie bereits erwähnt, die Tatsache, daß sich Caraccioli unter den Papstwählern befand und deshalb keine Aufgaben außerhalb des Konklaves wahrnehmen konnte. Unter Conzié standen Ugo de Robertis, Patriarch von Jerusalem, Giovanni Capogallo, Bischof von Novara, und Philibert de Naillac, seit 1395 Großmeister des Johanniterordens. Unter Androhung der Todesstrafe durfte sich niemand dem Palast auf weniger als 50 Ellen nähern, um die Kardinäle nicht zu beeinflussen. Dafür sorgten in erster Linie die Ritter des Johanniterordens, die in den Gärten und im Innenhof des Palastes Zelte aufgeschlagen hatten $^{83}$. Schon in der 17. Sitzung, am 13. Juni, schworen die Kardinäle, nur einen solchen zu wählen, der mindestens zwei Drittel der Stimmen beider Kollegien besaß. Nach nur elf Tagen einigte man sich (einstimmig!) auf den Kardinal von Mailand, Pietro Philargi (auch Petros Philargis oder Petrus de Candia), der unter dem Namen Alexander V. die Nachfolge der abgesetzten Päpste antrat $^{84}$. In der 20. Sitzung des Konzils (1. Juli 1409) übernahm Alexander den Vorsitz und ließ durch Baldassare Cossa (Kardinaldiakon von Sankt Eustachius und päpstlicher Legat in Bologna) mehrere Dekrete verlesen, nachdem er zuvor seine kurialen Beamten ernannt und bestätigt hatte ${ }^{85}$. Gerade dieser Punkt bedarf genauerer Untersuchung. Jedes Amt des päpstlichen Hofes war während des Schismas doppelt besetzt worden. Es gab zwei Päpste, zwei Schatzmeister, zwei Kanzler und selbstverständlich auch zwei Kämmerer (Kardinal Caraccioli und François de Conzié). Wer sollte nun welchen Posten erhalten? Der neue Papst hatte sich zu entscheiden und mußte strukturelle Änderungen und die damit verbundene Ämterverteilung möglichst rasch vorneh-

${ }^{81}$ SOUCHON, Papstwahlen (wie Anm. 27), S. 55, 60.

${ }^{82}$ Ibid., S. 61.

${ }^{83}$ Ibid.

${ }^{84}$ Chronique du religieux (wie Anm. 17), lib. XXX, S. 4.

${ }^{85}$ HEFELE, Conciliengeschichte (wie Anm. 15), Bd. 6, S. 1033. 
men. Die Wahl für das Amt des apostolischen Kämmerers fiel auf Conzié. Dafür gab es mehrere Gründe. Zum einen war er der Erfahrenere von beiden, wenn man bedenkt, daß Conzié 1383, Caraccioli hingegen erst 1404 ernannt worden war $^{86}$. Mit der längeren Amtszeit stand Conzié auch in einer ganz anderen Tradition. Da er kein Kardinal war, konnten keine Diskussionen darüber aufkommen, wer ihn dazu ernannt hätte. Caraccioli hingegen wurde 1405 von Innozenz VII. kreiert, was im Falle von dessen Emennung den neuen Papst eindeutiger in die Tradition der römischen Obödienz setzen würde. Abgesehen davon konnte Alexander mit Conzié als Kämmerer die alte Regel, daß ein Kämmerer nicht Kardinal sein dürfe, neu beleben.

Ein weiterer Vorteil Conziés gegenüber seinem ehemals römischen Amtskollegen war dessen Fachwissen auf dem zeremoniellen Gebiet, wenn man die eigenen Schriften Conziés berücksichtigt ${ }^{87}$. Es ist anzunehmen, daß besonders etwaige Schützlinge Conziés und Männer, die er selbst zu seinen Mitarbeitern ernannt hatte, zusammen mit dem Kämmerer den Anschluß an Alexander V. fanden $^{88}$.

Das Konzil endete am 7. August 1409 mit der 23. Sitzung. Ursprünglich wollten die Kardinäle nicht nur das Schisma beenden, sondern auch eine Kirchenreform vollziehen. Letzteres geriet allerdings rasch ins Stocken, worauf Alexander V. festlegen ließ, daß nach drei Jahren ein weiteres Konzil stattfinden solle, das sich mit der Reformation der Kirche befassen würde. Der Papst blieb noch bis November 1409 in der Stadt. Anfang November schickte Alexander V. Kardinal Thury und den apostolischen Kämmerer an den französischen Hof. Letzterer sollte noch ausstehende Abgaben des Königreiches einfordern und der Kardinal unterstützte ihn bei dieser Aufgabe ${ }^{89}$. Mitte November brach eine Seuche in Pisa aus und der Papst verließ die Stadt, um nach Pistoia zu gehen. Dort erfuhr er, daß es seinen Kämpfern am 13. Dezember gelungen war, Rom zu nehmen. Besonders die römische Stadtbevölkerung forderte nun die "Rückkehr» des Papstes in die Heilige Stadt, doch Kardinal Cossa empfahl Alexander, nicht nach Rom zu gehen, sondern sich in Bologna niederzulassen. Hatte das Konzil seine Gegner (Benedikt und Gregor) bereits vor der Wahl Alexanders mit einer Bulle verdammt, erneuerte dieser den Bannstrahl am 31. Januar 1410 von Bologna aus und bestätigte damit die Beschlüsse der Kirchensynode von Pisa ${ }^{90}$. Kurze Zeit später erschien eine Gesandtschaft der Stadt Rom, die ihm die Schlüssel der Stadt überbrachte und ihn bat, sich doch dorthin zu begeben. Der Papst aber zögerte zu lange mit seiner Antwort und verstarb wenige Wochen nach der Ankunft der Abordnung

\footnotetext{
${ }^{86}$ SouCHON, Papstwahlen (wie Anm. 27), S. 279.

${ }^{87}$ Le Diare de François de Conzié (Anm. 51), S. 109.

${ }^{88}$ BINZ, Népotisme (wie Anm. 2), S. 111.

${ }^{89}$ ValoIS, La France (wie Anm. 12), Bd. 4, S. 178.

${ }^{90}$ HEFELE, Conciliengeschichte (Anm. 15), Bd. 7, S. 3.
} 
am 3. Mai 1410. Noch am Totenbett soll er die Rechtmäßigkeit der Pisaner Beschlüsse beteuert und die Kardinäle zur Einheit ermahnt haben ${ }^{91}$.

Wie bereits erwähnt, mußte Alexander V. unmittelbar nach seiner Wahl Entscheidungen über die neue Ämterverteilung vornehmen, da viele kuriale Stellen doppelt besetzt waren. Da sich zwei Kämmerer in Pisa einfanden, hatte er zwischen Kardinal Caraccioli und François de Conzié zu entscheiden. Der Papst ernannte den Kardinal zum Generalvikar von Avignon und dem Comtat Venaissin und behielt Conzié als apostolischen Kämmerer. Nachdem Caraccioli jedoch verstarb (15. Februar 1411), ohne sein neues Amt angetreten zu haben, berief er Kardinal Thury zu dessen Nachfolger. Die Stadt und der Comtat waren bis zur Wahl Alexanders V. in der Obödienz Benedikts XIII. geblieben. Dieser hatte noch vor seiner Abreise nach Savona seinen Neffen Rodrigo de Luna zum Gouverneur der Stadt Avignon und des Venaissins ernannt und ihm befohlen, den päpstlichen Palast in Avignon und andere Posten der Stadt zu befestigen, um sicherzustellen, daß ihm Avignon erhalten bleiben würde. Zusammen mit dem Vicomte de Vols, der zum Militärkommandanten bestimmt wurde, rüstete Luna auf und holte eine große Anzahl aragonesischer und katalanischer Soldaten in die Stadt und in den Comtat ${ }^{92}$. Um die spanischen Truppen im Papstpalast zu bezwingen, lieh sich der Kardinal Bombarden und Katapulte der Städte Carpentras und Aix (die grande bombarde von Aix mußte von 36 Pferden gezogen werden und gehörte wohl zu den größten ihrer Zeit). Am 9. Dezember 1410 starb Kardinal Thury und die Belagerung hielt immer noch an, als Johannes XXIII. (der Nachfolger des mittlerweile verstorbenen Alexander V.) François de Conzié zu seinem Generalvikar in Avignon und der Grafschaft Venaissin ernannte. Im Januar 1411 kam Conzié in der Stadt an, wo sich Luna weiterhin gegen die Belagerung zur Wehr setzte. Conzié ließ den Kriegsrat zusammentreten und setzte den Termin für den endgültigen Angriff auf Rodrigo de Luna für den 8. Februar 1411 fest. Nachdem die dafür benötigten neuen Hilfstruppen aus dem Venaissin und Frankreich noch nicht eingetroffen waren, konnte der Termin nicht eingehalten werden und der große Angriff fand mit hohen Verlusten auf beiden Seiten am 14. Februar 1411 statt. Die Belagerer griffen an drei Stellen des Palastes gleichzeitig an. Doch selbst diese heftige Offensive führte keineswegs zur Kapitulation des Neffen Benedikts XIII. ${ }^{93}$. Dennoch resultierte daraus die beidseitige Einsicht, daß man verhandeln mußte, um den Kampf zu beenden. Nach zahlreichen Verhandlungen wurde ein halbes Jahr später, am 30. September 1411, ein Vertrag zwischen den beiden Lagern unterzeichnet. Für die Seite der Belagerten siegelten Rodrigo de Luna und der Vicomte de Vol, für die

\footnotetext{
${ }^{91}$ Chronique du Religieux (Anm. 17), lib. XXXI, S. 7.

92 Joseph FORNERY, Histoire du Comté Venaissin et de la ville d'Avignon, Avignon 1910, Bd. 1, S. $417 f$.

${ }^{93}$ Valois, La France (wie Anm. 12), Bd. 4, S. 162.
} 
Belagerer François de Conzié, Jean de Poitiers und Constantin de Pergula, ein Sekretär Johannes' XXIII. Den Belagerten wurde gestattet, drei Männer auszuwählen, die sich mit je einem Knappen zu Pedro de Luna (Benedikt XIII.) begeben durften, um ihm vom Zustand des Palastes und von der ganzen Situation zu berichten. Wenn sie innerhalb einer fünfzigtägigen Frist nicht zurückkommen würden, um weiterzukämpfen, müßten die verbleibenden Männer abziehen, den Palast dem päpstlichen Generalvikar, François de Conzié, und die Festung Oppède dem päpstlichen Rektor, Jean de Poitiers, aushändigen ${ }^{94}$.

Am 22. November 1411 war die Frist verstrichen, ohne daß Benedikt Hilfstruppen zur Unterstützung seines Neffens geschickt hatte. Wie vertraglich festgelegt, verließen die spanischen Truppen Lunas sowohl Avignon als auch das Venaissin ${ }^{95}$.

\footnotetext{
${ }^{94}$ FORNERY, Histoire (wie Anm. 92), Bd. 1, S. 427.

${ }^{99}$ VAlO1S, La France (wie Anm. 12), Bd. 4, S. 170 f.
} 
